\title{
ANCHORS: an Archive of Chandra Observations of Regions of Star formation
}

\author{
Scott J. Wolk and Bradley D. Spitzbart \\ High-Energy Division, Harvard-Smithsonian Center for Astrophysics, \\ 60 Garden Street, Cambridge MA 02138, USA \\ email: swolk,bspitzbart@head.cfa.harvard.edu
}

ANCHORS is a web based archive of all the point sources observed during Chandra observations of regions of star formation. It is designed to aid both the X-ray astronomer with a desire to compare X-ray datasets and the star formation astronomer wishing to compare stars across the spectrum. For some 50 Chandra fields, yielding $10000+$ sources, the database contains X-ray source properties including position, net count rates, flux, hardness ratios, lightcurve statistics and plots.

Spectra are fit using several models, with final parameters and plots recorded in the archive. Multi-wavelength images and data are cross-linked to other archives such as 2MASS and SIMBAD. The pipeline processing ensures consistent analysis techniques for direct comparisons among clusters. Results are presented on-line with sorting, searching, and download functions HTML/XML interface. We are hoping to add linkage to the VO. We will solicit users' feedback.

\section{End-to-end science from major facilities: does the $\mathrm{VO}$ have a role?}

\author{
Gerard F. Gilmore \\ Institute of Astronomy, University of Cambridge, Madingley Road, Cambridge, CB3 0HA, UK \\ email: gil@ast.cam.ac.uk
}

The Virtual Observatory provides a natural solution to the existence problem in communications: how can one ask a question of another unless you know the other exists? Many think e-mail from apparent strangers, e-blogs, etc., suggest there is no shortage of possible such solutions. In that context, is the Virtual Observatory in fact the necessary and desirable part of the solution? Specifically, does the VO necessarily play a critical role in delivering end-to-end facility science, from ideas, through proposals, resources/ facilities, to distributed, reviewed, knowledge? If not, what else needs to be added? 